May 2007

\title{
How Black Holes Form in High Energy Collisions
}

\author{
Nemanja Kaloper1 ${ }^{1}$ and John Terning 2 \\ Department of Physics, University of California, Davis, CA 95616
}

\begin{abstract}
We elucidate how black holes form in trans-Planckian collisions. In the rest frame of one of the incident particles, the gravitational field of the other, which is rapidly moving, looks like a gravitational shock wave. The shock wave focuses the target particle down to a much smaller impact parameter. In turn, the gravitational field of the target particle captures the projectile when the resultant impact parameter is smaller than its own Schwarzschild radius, forming a black hole. One can deduce this by referring to the original argument of escape velocities exceeding the speed of light, which Michell and Laplace used to discover the existence of black holes.
\end{abstract}

\footnotetext{
${ }^{1}$ kaloper@physics.ucdavis.edu

${ }^{2}$ terning@physics.ucdavis.edu
} 
Quantum gravity has long been a holy grail of fundamental theory. The quest for it has been slow and arduous, in no small part due to an acute lack of experimental milestones that could point the way. Until recently this seemed inevitable, due to the unbearable feebleness of gravity. However the advent of the braneworld paradigm has rekindled some hopes that nature might be kinder to us. Perhaps gravity is stronger at sub-mm distances, giving the prospects that in some future collider experiments effects of quantum gravity might be directly encountered. If the fundamental scale of gravity is really low, near a $\mathrm{TeV}$, such effects might be within the reach of LHC [1]. Among the possible direct signatures, certainly the most dramatic phenomena may be the formation of black holes in high energy collisions, and their subsequent decay by Hawking radiation. This interesting possibility has attracted a lot of attention from theorists and experimentalists alike, spurring a great deal of exploration of the likelihood and signatures of such processes, as exemplified in [2].

The exact description of black hole formation in a collision of real particles does not yet exist. As a result, the analyses of black hole formation processes [2] estimate the black hole production cross section by the horizon area of a black hole whose horizon radius $r_{h}$ is set by the center-of-mass collision energy $\sqrt{s}$. When the impact parameter $b$ is smaller than $r_{h}$ it is assumed that the efficiency of formation of a black hole is close to $100 \%$. The strongest support for this comes from examining the gravitational shock wave fields of zero rest mass particles [3, 4, 5], and seeing when a trapped surface forms as the shock waves cross. These analyses, in particular the work of Eardley and Giddings [5] which covers off-center collisions with $b>0$, find that trapped surfaces form when $b \lesssim r_{h}$, and have the area scaling as the horizon area $\sim r_{h}^{2} \sim G_{N}^{2} s$, where $r_{h}$ is the horizon radius and $G_{N}$ is Newton's constant. However the shock wave solutions inevitably break down when the fields of different particles cross. Since the black hole cross sections are extracted from the shocks, a priori they remain sensitive to the nonlinear corrections, which still obscures the interpretation of the results.

On the other hand, the cross section estimates by the horizon area for off-center collisions were disputed in [6]. In these papers, Voloshin has eloquently argued that the black hole formation cross section should be exponentially suppressed for off-center collisions, by the exponent of the Euclidean action of the black hole. In a nutshell, the main tenet of this argument was that at very high energies the direct local interactions of particles must be strongly suppressed because of Lorentz boosts. At high speeds, the particles occupy small regions of space $\lambda \sim 1 / p \ll b$, in the units $c=\hbar=1$. Thus, the argument goes, they don't get to interact strongly unless the collisions are head-on, and so black hole formation cannot be a local process. Instead, contends [6], the description of black hole formation must be based on some kind of instanton solution, which would give an exponential suppression whenever $b>0$.

Given these doubts one might be led to consider an extreme case: a frame of reference where one particle is almost at rest and the other is highly boosted. The highly boosted particle is nothing like a black hole and the slowly moving particle at a large impact parameter is a tiny perturbation. So, how do black holes really form in collisions? We will describe a very simple and intuitively clear physical picture whereby a black hole forms in a classical capture process. This is why the estimate of black hole formation cross section by the classical horizon area is the correct thing to do, as argued by [3, 4, 5], and why the nonlinear corrections should not change it qualitatively. To see this, it is enough to use 
a)

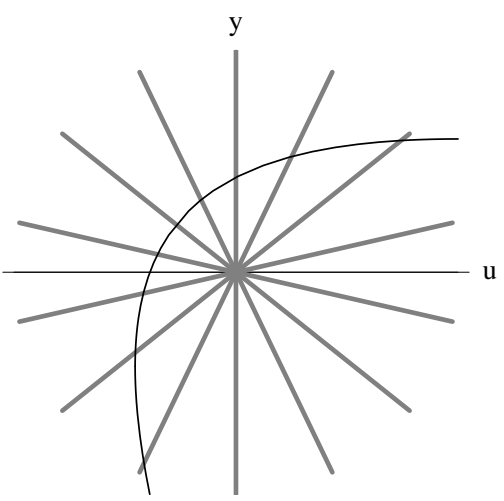

b)

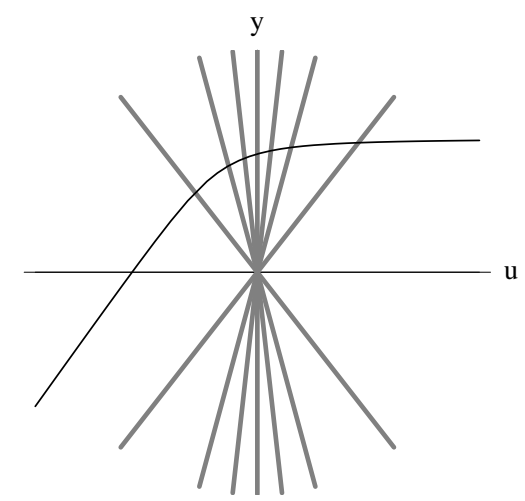

c)

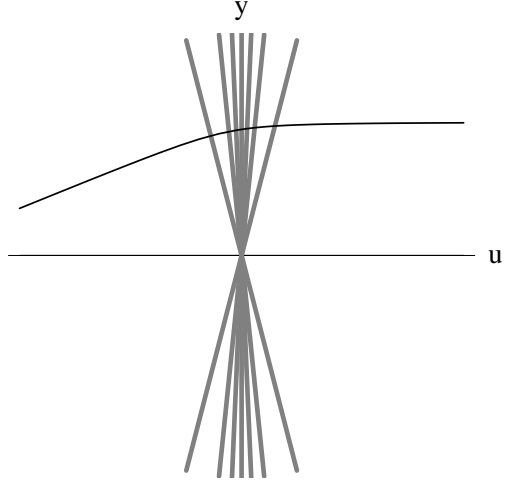

Figure 1: Scattering trajectories of a slowly moving electric charge by a) a stationary charged particle, b) a charged particle moving at $v=0.94 c$, and c) a charged particle moving at $v=0.99 c$. The gray lines indicate the electric field in each case. With the moving particle going along the $z$ axis, the coordinate $u=z-v t$, so this particle is always at $u=0$. The charges are not held fixed here.

the original, simple escape velocities argument, that led John Michell [7] in 1784 and Pierre Simon, Marquis de Laplace [8] in 1796 to deduce the existence of black holes, some 130 years before the inception of General Relativity. The details of trapped surface formation are not essential here, although they may be necessary for comparison with precision black hole experiments.

The situation we are considering is entirely analogous to the electromagnetic scattering of a slowly moving charge by a rapidly moving one as shown in Fig. 1. When a charge is highly boosted its electric field lines are compressed into the directions transverse to its motion. Most of the scattering of a slow charge takes place while it moves through this region with a more intense field. Away from it the slow charge experiences almost no force.

Now consider what happens in gravitational scattering. In the limit of an infinite boost all of the gravitational field is confined inside a transverse plane, which is known as a gravitational shock wave [9]. The metric around the shock wave is just two patches of flat space sewn together, so it is not surprising that the geodesics around a shock wave found by Dray and 't Hooft [10] correspond to particles travelling freely except when they cross the shock wave front. The scattering angle can easily be computed, but qualitatively we know that the amount of bending will be proportional to the momentum of the highly boosted particle 
since that is its gravitational 'charge'. Thus for a large enough boost we can get scattering at almost right angle in the $u-y$ plane, and send a particle with a large impact parameter onto a scattered trajectory that passes very close to the path of the boosted particle. If the scattered particles end up within a distance smaller than the horizon of the target particle then we certainly expect a black hole to form.

Let us now elaborate our argument. We will specialize to the case of four dimensions since it illustrates the issues in the simplest way possible. Consider a collision of two massive particles with rest masses $m$ and $M$, which move towards each other with relative (and relativistic!) velocity $\overrightarrow{\mathrm{v}}$, and impact parameter $b$. When they are far away from each other, as long as they are massive, we can always go to the rest frame of one of them, say $M$. At large relative velocities, $\mathrm{v} \rightarrow 1$, the gravitational field of the particle $m$ is extremely strongly boosted in the rest frame of the particle $M$. Most of the field is being carried by the particle momentum $p \sim E=m / \sqrt{1-\mathrm{v}^{2}}$, by the equivalence principle. In this limit, we can approximate the field by the linearized Schwarzschild solution, boosted to a very large velocity $\overrightarrow{\mathrm{v}}$. The nonlinear corrections to the field can be organized as an expansion in $m / p$, such that in the infinite boost limit, where we also take $m \rightarrow 0$ and hold $p$ fixed, the metric reduces to an exact shock wave metric. In $4 D$, the shock wave fields are given in [9, 10], and can be generalized beyond $4 D$ [11] and to codimension-2 tensional branes [12].

In the infinite boost limit, when the source has zero rest mass, the shock wave field is completely confined in the null transverse surface orthogonal to the direction of motion, anchored at the instantaneous location of the particle. For fast particles of nonzero rest mass, the shock wave approximation breaks down far away from the moving particle, because the field lines will spread out of this surface. This will occur at transverse distances from the source which are of the order of $\ell \sim r_{h}(m) / \sqrt{1-\mathrm{v}^{2}}$. Beyond this distance, we can't neglect the spreading of the field lines in the direction of motion, because an observer farther away than $\ell$ will see the effects of retardation due to the slightly sub-luminal motion of the source in the limit $\mathrm{v} \rightarrow 1$. Conversely, whenever the impact parameter $b$ of the collision is smaller than $\ell$, we can use the shock wave field to extract the information about the black hole formation to the leading order in $m / p$.

We can estimate how far from the source we can trust the shock wave approximation in practice. First, we have $1 / \sqrt{1-\mathrm{v}^{2}} \sim p / m$. Next, the gravitational radius $r_{h}$ of a particle and its mass are related through the Schwarzschild solution by $r_{h}=2 G_{N} m$. Using this, the scale $\ell$ out to which we can use the shock wave approximation for the field of the fast particle is

$$
\ell \sim G_{N} p \sim \frac{p}{8 \pi M_{4}^{2}}
$$

where we have defined the $4 D$ Planck mass $M_{4}^{2}=\frac{1}{8 \pi G_{N}}$. Thus for impact parameters of the order of $b \ll \ell$ the shock wave is an excellent approximation. Clearly, we also need to require that $b>1 / M_{4}$ in order to trust the classical description in the first instance.

To write the shock wave solutions, we can use the simple cutting-and-pasting technique of [10]. Instead of the lightcone coordinates, we will use the standard Minkowski coordinates to simplify the presentation. We take the null line $u=\frac{t-z}{2}=0$ as the trajectory of the projectile, and introduce a discontinuity in the orthogonal null coordinate $v=-\frac{z+t}{2}$ by replacing $d v$ in the metric by $d v-f(x, y) \delta(u) d u$ [10]. Here $f$ is the shock wave profile in the 
transverse null plane anchored to the instantaneous location of the particle, and $x, y$ denote the spatial dimensions along it. The metric is, in Minkowski coordinates,

$$
d s_{4}^{2}=d x^{2}+d y^{2}+d z^{2}-d t^{2}+2 \delta(z-t) f(x, y)(d z-d t)^{2} .
$$

The shock wave profile $f(x, y)$ is the solution of the Poisson equation in the transverse plane $\nabla_{2}^{2} f=\frac{2 p}{M_{4}^{2}} \delta(x) \delta(y)$, which arises from imposing the Israel junction condition on the wave front. This means that $f$ is the $2 \mathrm{D}$ Coulomb potential of a 'charge' $p$ at the origin,

$$
f=\frac{p}{\pi M_{4}^{2}} \ln \left(\frac{\sqrt{x^{2}+y^{2}}}{L}\right),
$$

where $L$ is an arbitrary integration constant [9, 10].

The shock wave metric (2) shows that the gravitational interaction - as imparted by the field of the fast particle - between our two colliding particles is practically instantaneous, occurring at the moment when the shock front crosses the particle $M$. Before and after this, the particle $M$ moves inertially. Before the collision its gravitational field will deflect the projectile, but this deflection angle, $\Delta \simeq \frac{G_{N} M}{b}$, will be sufficiently small to be neglected when $b \gg G_{N} M$. This justifies our choice of the initial rest frame of $M$ as the stage on which to set up our analysis, and our neglecting the field of $M$ before the collision.

It is now straightforward to see what happens when a particle $M$ encounters the shock wave field (2). It will not remain at rest, because at the moment the shock passes by it, it will be strongly attracted towards the source of the shock wave. As long as the relative velocity $\mathrm{v}$ is very close to the speed of light, the interaction is impulsive, and the shock wave behaves as a very thin gravitational lens. The total physical deflection is Lorentz-invariant, and could be computed (with more difficulty) in some other frame. The observed deflection angle, however, needs to be properly transformed according to Lorentz transformation rules, in order to account for relativistic aberration between different inertial observers. It is now easy to solve for the timelike geodesics of the shock metric (2) to find the spacetime trajectory of the particle $M$, at rest before the collision. Since the problem is central, we can orient it so that the motion is in the $x=0$ plane, by taking the initial impact parameter to be along the $y$ direction. Then the trajectory of the target is

$$
y=b-\Theta(\tau) \mathrm{v}_{f} \tau, \quad z=\frac{1}{2} \Theta(\tau) \mathrm{v}_{f}^{2} \tau-\Theta(\tau) f(0, b), \quad t=\tau+\frac{1}{2} \Theta(\tau) \mathrm{v}_{f}^{2} \tau-\Theta(\tau) f(0, b),
$$

where $\tau$ is the proper time of the particle $M, \Theta(\tau)$ is the Heaviside step function, $b$ is the impact parameter, and $\mathrm{v}_{f}=\left.\partial_{y} f\right|_{y=b}=\frac{p}{\pi M_{4}^{2} b}$ is the final 4-velocity component of $M$ in the $y$ direction. The collision occurs at $\tau=0$, and the post-collision 4 -velocity is set by the impulsive force pulling $M$ towards the projectile at the moment of wave front crossing. Similarly, the projectile moves along

$$
y=0, \quad z=t=\tau+\frac{1}{2} \Theta(\tau) \mathrm{v}_{f}^{2} \tau-\Theta(\tau) f(0, b),
$$

where the parameterization of the solution is chosen to synchronize the projectile with the target clock before the collision. 
Now, in the frame where it was initially at rest, the target particle $M$ after the collision may end up moving very fast. This will happen for impact parameters $b \ll p / M_{4}^{2}$. For greater impact parameters, the target will move slowly, and nothing dramatic will happen, but as we will see that case is irrelevant for black hole formation. We can find the postcollision coordinate velocities of the mass $M$ in the $y$ and $z$ directions from the post-collision trajectory (4),

$$
\mathrm{v}_{y}=\frac{d y}{d t}=-\frac{\mathrm{v}_{f}}{1+\frac{\mathrm{v}_{f}^{2}}{2}}, \quad \mathrm{v}_{z}=\frac{d z}{d t}=\frac{\frac{\mathrm{v}_{f}^{2}}{2}}{1+\frac{\mathrm{v}_{f}^{2}}{2}},
$$

so that after the collision, the speed of $M$ in the frame where it was originally at rest is $\mathrm{v}_{M}=\sqrt{\mathrm{v}_{y}^{2}+\mathrm{v}_{z}^{2}} \rightarrow 1$, when $\mathrm{v}_{\mathrm{f}}=\frac{p}{\pi M_{4}^{2} b} \gg 1$. However, Eq. (6) reveals that this is because after crossing the shock wave, the target began moving very rapidly in the $z$-direction, chasing after the projectile. The velocity of the target particle $M$ in the $y$ direction remains very small. The shock pulls the target along, accelerating it to a very high velocity almost parallel with the shock source.

To see what happens next, we switch to a new inertial frame, boosting in the $z$ direction to cancel the $\mathrm{v}_{z}$ component of the target post-collision velocity. We can always do this after the collision, since the shock wave field has moved ahead and the target is chasing after it in the vacuum left in the projectile's wake. In this new frame the target moves very slowly in the $y$ direction, and the spatial separation between the two particles is $r^{2} \simeq(\Delta y)^{2}+\tau^{2}$, where $\Delta y$ is the transverse distance between the particle trajectories after the collision and $\tau$ is the distance that the fast particle travelled after the collision, moving at near the speed of light. This is because $\tau$ is the proper time of the target, as can be checked from the solution (4), and since the target is moving very slowly the difference between the coordinate time and proper time is minuscule. Using our solutions and the expression for $\mathrm{v}_{f}$, this yields $r^{2} \simeq b^{2}\left(1-\frac{p}{\pi M_{4}^{2} b} \frac{\tau}{b}\right)^{2}+\tau^{2}$. It is simple to see that this is minimized when $r^{2} \simeq \tau_{*}^{2}$, where the time

$$
\tau_{*}=\frac{\pi M_{4}^{2} b^{2}}{p}
$$

is the instant when the particle $M$ will pass through $x=y=0$.

While the target particle $M$ won't feel the gravitational effects of the shock wave field any more, the projectile will have to work against the gravitational attraction of the target in order to continue fleeing away. If it is to escape away to infinity, it must move faster than the escape velocity set by the field of the target particle at a distance $\sim \tau_{*}$ from it. Since in this frame the target is again slow, we can estimate its gravitational pull on the projectile by its Newtonian potential at the minimal separation between the particles after the collision. This yields $V_{\text {escape }}^{2} \simeq \frac{M}{M_{4}^{2} \tau_{*}}$ for the escape velocity at a distance $r=\tau_{*}$ from $M$. Substituting Eq. (17) and noting that $M p \simeq s$, we finally obtain

$$
V_{\text {escape }}^{2} \simeq \frac{s}{M_{4}^{4} b^{2}}
$$

Now, exactly as Michell [7] and Laplace [8] first noted, the projectile will be unable to escape 
if $V_{\text {escape }} \geq 1$. Hence whenever the initial impact parameter satisfies

$$
b \leq \frac{\sqrt{s}}{M_{4}^{2}}
$$

the target will capture the projectile. In other words after being focused by the shock wave the separation of the two particles is $\tau_{*}<r_{h}(M)$, so a black hole must form. As a check note that when $p \gg M, \sqrt{s} \ll p$, and so indeed $b \ll \ell$, which therefore means that our approximations of the projectile gravitational field by a shock wave are fully justified. Further, in this limit $\mathrm{v}_{f} \gg 1$, so our boosting to the new post-collision frame is consistent. It is also worth noting that from Eq. (6) $v_{y}=\pi \sqrt{s} / p \ll 1$ where we have used the maximal value of $b$ from Eq. (9). Thus even after crossing the shock wave the target particle is still travelling slowly in the $y$ direction unless the initial impact parameter was much smaller that the typical value for black hole formation. Moreover, imposing $b>1 / M_{4}$ we find from Eq. (9) that for black hole to form we must ensure $s>M_{4}$. Hence the center-of-mass energy for black hole formation must be trans-Planckian. Then, the cross section for the black hole to form is given by the final black hole area,

$$
\sigma_{\text {black hole }} \simeq \pi b_{\text {critical }}^{2} \simeq \frac{s}{M_{4}^{4}} .
$$

This completes our argument.

We conclude that indeed the estimates of the black hole production cross section by the classical center-of-mass horizon area are justified. In a frame where one of the particles starts at rest, the process of black hole formation looks like the capture of the projectile by the target particle, after the target is focused down to a very small impact parameter by the shock wave field of the projectile. For precision calculations, one may still need to use the more involved derivations of the formation of trapped surfaces in the collision of two shock waves. It would, however, be interesting to refine our argument and see how the results of a next-to-leading order treatment of the classical capture compare to the trapped surface formation criteria.

It would also be interesting to extend this reasoning to higher-dimensional examples and braneworld setups [13], which could be relevant for the forthcoming LHC searches.

We thank M. Park for useful discussions. NK and JT were supported in part by the DOE Grant DE-FG03-91ER40674. NK was also supported in part by a Research Innovation Award from the Research Corporation.

\section{References}

[1] N. Arkani-Hamed, S. Dimopoulos and G. R. Dvali, Phys. Lett. B 429, 263 (1998).

[2] P. C. Argyres, S. Dimopoulos and J. March-Russell, Phys. Lett. B 441, 96 (1998); T. Banks and W. Fischler, hep-th/9906038; R. Emparan, G. T. Horowitz and R. C. Myers, Phys. Rev. Lett. 85, 499 (2000); S. Dimopoulos and G. Landsberg, Phys. Rev. Lett. 87, 161602 (2001); S. B. Giddings and S. D. Thomas, Phys. Rev. D 65, 056010 (2002). 
[3] R. Penrose, unpublished, 1974.

[4] P. D. D'Eath and P. N. Payne, Phys. Rev. D 46, 658 (1992); Phys. Rev. D 46, 675 (1992); Phys. Rev. D 46, 694 (1992).

[5] D. M. Eardley and S. B. Giddings, Phys. Rev. D 66, 044011 (2002).

[6] M. B. Voloshin, Phys. Lett. B 518, 137 (2001); Phys. Lett. B 524, 376 (2002) [Erratumibid. B 605, 426 (2005)].

[7] J. Michell, Phil. Trans. Roy. Soc., 74, 35-57 (1784).

[8] Pierre-Simon, Marquis de Laplace, Exposition du système du Monde, 1796.

[9] P. C. Aichelburg and R. U. Sexl, Gen. Rel. Grav. 2, 303 (1971).

[10] T. Dray and G. 't Hooft, Nucl. Phys. B 253, 173 (1985); Class. Quant. Grav. 3, 825 (1986).

[11] V. Ferrari, P. Pendenza and G. Veneziano, Gen. Rel. Grav. 20, 1185 (1988); H. de Vega and N. Sanchez, Nucl. Ph. B 317, 706 (1989).

[12] N. Kaloper and D. Kiley, JHEP 0603, 077 (2006).

[13] N. Kaloper and J. Terning, work in progress. 